\title{
Suggesting a way to understand the actual potential of anti-Alzheimer's disease drugs that show promise in transgenic mouse models
}

\author{
Rafael Franco ${ }^{1,2 *}$ and Milos Petrovic ${ }^{3}$ \\ ${ }^{1}$ Departament de Bioquímica i Biologia Molecular, Facultat de Biologia, Universitat de Barcelona, Barcelona, Spain, ${ }^{2}$ Centro de \\ Investigación Biomédica en Red: Enfermedades Neurodegenerativas (CIBERNED), Instituto de Salud Carlos III, Madrid, Spain, \\ ${ }^{3}$ School of Pharmacy and Biomedical Sciences, University of Central Lancashire, Preston, UK
}

Keywords: chiral drug, memory, Alzheimer's disease, nootropic, neuroprotection, enantiomers

A commentary on

Effects of (-)epicatechin on the pathology of APP/PS1 transgenic mice

by Zeng Y-Q, Wang Y-J, Zhou X-F. (2014). Front Neurol 5:69. doi:10.3389/fneur.2014.00069

One conundrum in Alzheimer's disease (AD) research using transgenic mouse models is the high amount of successful memory-enhancing drugs. By contrast, very few drugs and of limited efficacy are available for humans having this pathology. As previously discussed (1), the advance in this field, i.e., to fulfill the translational facet of anti-AD research, requires deciphering why so many different drugs (or therapeutic interventions, such as exercise or training) have memory-enhancing properties in transgenic models of the disease. Transgenic animals do not accurately reflect the human disease, as they overexpress proteins with mutations that appear only in a reduced percentage of patients (2). The majority of patients have late-onset clinical symptoms due to multiple factors many of which may be circumstantial. On waiting for the development of novel animals models that may, eventually, shorten the distance between the lab bench and the bedside (3), we should take advantage of the huge amount of data showing promise of different drugs in transgenic models. A way to do it is by designing medium-to-high throughput experiments to compare anti-AD effects of closely related drugs. In this commentary, we focus on small drugs with the same chemical formula, but with different 3-D structure.

A significant number of drugs approved for human consumption for fairly different illnesses have a special structural characteristic called stereoisomerism (see Figure 1). Examples of small molecules with alternative stereoisomer variants (enantiomers) that are already marketed for human consumption include verapamil, ibuprofen, citalopram, and thalidomide. Due to the difficulties in isolating the two enantiomer species and other operational reasons, mixtures of the two species are approved for human consumption.

\section{Verapamil}

Verapamil, the calcium channel blocker indicated in a variety of cardiovascular ailments, is the mixture of two enantiomers: $( \pm)$-2-(3,4-dimethoxyphenyl)-5-\{[2-(3,4-dimethoxyphenyl)ethyl](methyl)amino\}-2-prop-2-ylpentanenitrile. Despite the higher efficacy of the levo isomer, already reported in 1985 for atrioventricular conduction (4), the drug is still marketed as a mixture of levo and dextro species. The potential of verapamil in $\mathrm{AD}$ was assayed 18 years ago under the strategy of discovering new uses for drugs already approved for human consumption. 


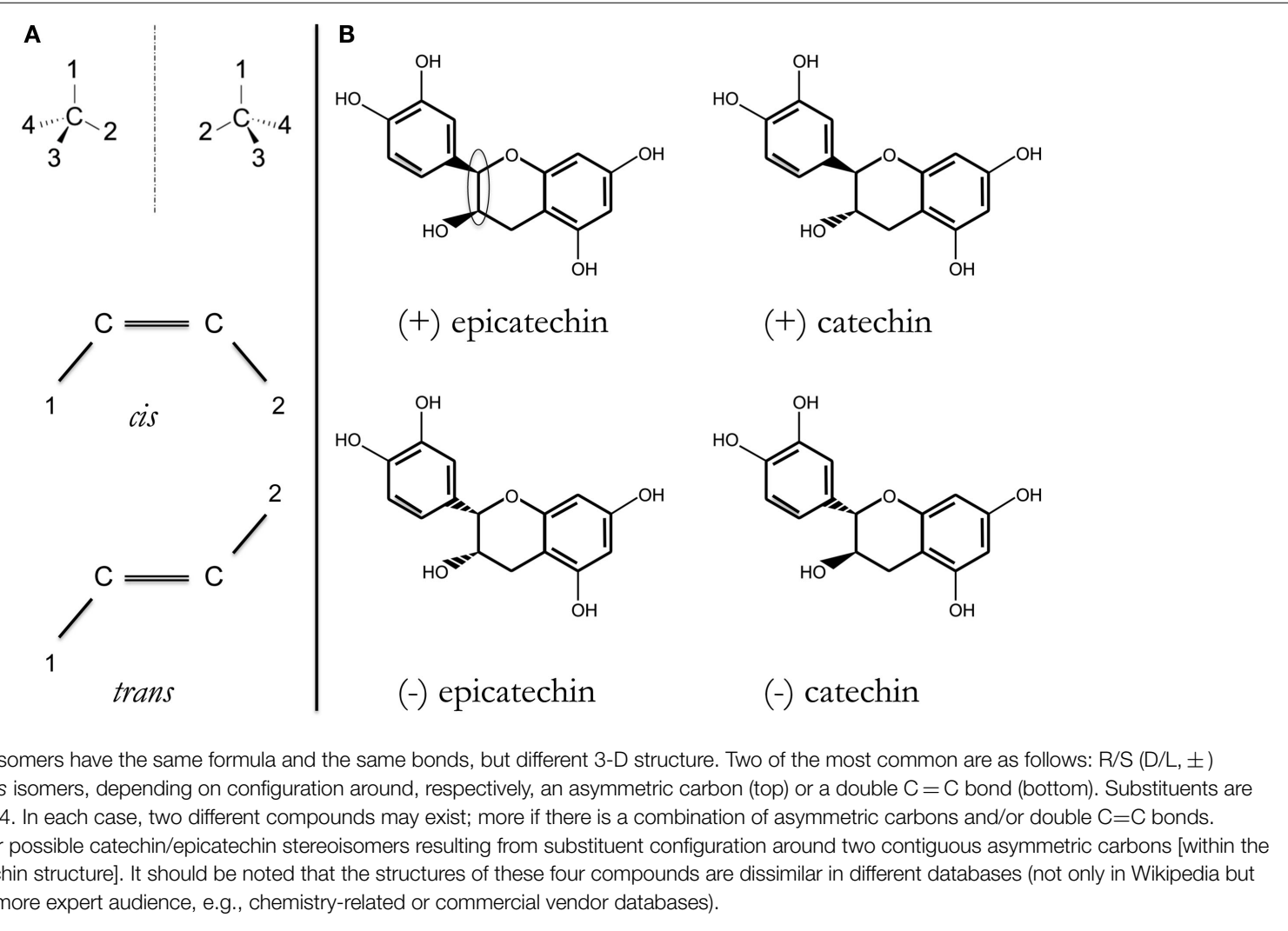

As verapamil ameliorates cognitive and non-cognitive deficits (5), we propose to test separately the levo and dextro verapamil molecules in $\mathrm{AD}$ models.

\section{Ibuprofen}

Controversy surrounds the usefulness of anti-inflammatory drugs in $\mathrm{AD}$ and one example is provided by ibuprofen, which suppresses plaque pathology in the Tg2576 AD model (6), while not improving deficits in the 5XFAD AD model (7). To our knowledge, the two components of ibuprofen $[( \pm) 2-(4-$ Isobutylphenyl)propanoic acid] have not been tested separately.

\section{Thalidomide}

Thalidomide, the leprostatic and sedative drug with cognitionenhancement and anti-amyloid $\beta(\mathrm{A} \beta)$ properties in mice models $(8,9)$, is constituted of two enantiomers $[( \pm)-2-(2,6$-dioxo3 -piperidinyl)-1H-isoindole-1,3(2H)-dione] that have not been individually tested in AD models.

Would it be convenient to test the different stereoisomers individually in $\mathrm{AD}$ models? Indeed, living animals are asymmetric at the macroscopic and at the molecular level (e.g., mammalian proteins are built up of $\mathrm{L}^{-}$, but not $\mathrm{D}$-amino acids), and, therefore, it is predictable that one stereoisomer may be more efficacious than the other(s).

Two enantiomers will likely have differential pharmacokinetics, differential metabolism, and differential mode of action (see Ref. (10) for recent review). Therefore, it is improbable that two enantiomers have similar - beneficial - effects in AD models, otherwise their action would be a general chemical one (for instance anti-oxidant, as discussed below) and not due to a specific mode of action. If the mode of action of a given asymmetric drug is specific, i.e., via inhibiting an enzyme or interacting with a receptor, chances of stereoisomers having similar in vivo potency are scarce. In that sense, one enantiomer may be the negative control of the other. One further concern in validating data from transgenic $\mathrm{AD}$ models is the usual finding of multiple beneficial effects of a given drug (e.g., improving spatial memory, decreasing $A \beta$ burden, reducing tau hyperphosphorylation). A compound having many benefits should be the exception and not the rule. Accordingly, scientists may consider which property is under study (behavioral, biochemical, etc.) and select the appropriate experimental model. It would also be desirable to determine the pharmacokinetics of the promising drugs to establish whether the individual enantiomers reach the brain at physiologically relevant concentrations or not

\section{Citalopram}

Escitalopram is one of the few examples of single stereoisomers that have reached the market. Citalopram (sold in different countries as: Celexa, Seropram, Talpram, Prisdal, Zentius, Cipramil, or generic citalopram) is one of the best-seller CNS drugs. Citalopram contains the racemic mixture of $\mathrm{R} / \mathrm{S}$ (or \pm ) 1-[3-(dimethylamino)propyl]-1-(4-fluorophenyl)-3H-2benzofuran-5-carbonitrile, but the S-enantiomer is better in inhibiting serotonin uptake and providing anxiolytic and antidepressant effects. Isolation of the $\mathrm{S}$ molecule led to the approval of 
escitalopram for clinical use (sold as Lexapro, Cipralex, or generic escitalopram). The difference between enantiomers is not a trivial one, as health and socio-economic benefits of escitalopram versus citalopram have been substantiated (11-14). Citalopram has been used in the Citalopram for Agitation in Alzheimer Disease Study (CitAD) randomized clinical with promising results (15). Thus, it would be reasonable to undertake another clinical trial to compare the effects of citalopram versus escitalopram in $\mathrm{AD}$ patients. Complementarily, it would be convenient to compare in animal $\mathrm{AD}$ models the efficacy of the two stereoisomers of citalopram in a variety of cognitive and molecular read-outs.

\section{Catechins}

Recently, substances with anti-oxidant properties have been found to be neuro-protective, indicating them as potential tools to combat AD. Polyphenols derived from plants and used in human nutrition may have anti-oxidant properties and neuroprotective potential. Let us consider close plant-derived compounds, tested in different experimental systems: $(+)$-catechin and (-)-epicatechin. One recent report has shown promise of $(-)$ epicatechin in transgenic AD models (16). However, in vitro, (+)catechin and $(-)$-epicatechin are equally efficacious in inhibiting formation of $A \beta$ fibrils from the precursor peptides $\left(A \beta_{1-40}\right.$ or $\mathrm{A} \beta_{1-42}$ ) (17). Is the anti-oxidant property of these compounds

\section{References}

1. Franco R, Cedazo-Minguez A. Successful therapies for Alzheimer's disease: why so many in animal models and none in humans? Front Pharmacol (2014) 5:146. doi:10.3389/fphar.2014.00146

2. Webster SJ, Bachstetter AD, Nelson PT, Schmitt FA, Van Eldik LJ. Using mice to model Alzheimer's dementia: an overview of the clinical disease and the preclinical behavioral changes in 10 mouse models. Front Genet. (2014) 5:88. doi:10.3389/fgene.2014.00088

3. Medina M, Avila J. The need for better AD animal models. Front Pharmacol (2014) 5:227. doi:10.3389/fphar.2014.00227

4. Echizen H, Vogelgesang B, Eichelbaum M. Effects of d,l-verapamil on atrioventricular conduction in relation to its stereoselective first-pass metabolism. Clin Pharmacol Ther (1985) 38(1):71-6. doi:10.1038/clpt.1985.137

5. Popović M, Caballero-Bleda M, Popović N, Bokonjić D, Dobrić S. Neuroprotective effect of chronic verapamil treatment on cognitive and noncognitive deficits in an experimental Alzheimer's disease in rats. Int J Neurosci (1997) 92(1-2):79-93. doi:10.3109/00207459708986392

6. Lim GP, Yang F, Chu T, Chen P, Beech W, Teter B, et al. Ibuprofen suppresses plaque pathology and inflammation in a mouse model for Alzheimer's disease. J Neurosci (2000) 20(15):5709-14.

7. Hillmann A, Hahn S, Schilling S, Hoffmann T, Demuth HU, Bulic B, et al. No improvement after chronic ibuprofen treatment in the 5XFAD mouse model of Alzheimer's disease. Neurobiol Aging (2012) 33(4):.e39-50. doi:10.1016/j. neurobiolaging.2011.08.006

8. Moreira AL, Wang J, Sarno EN, Kaplan G. Thalidomide protects mice against LPS-induced shock. Braz J Med Biol Res (1997) 30(10):1199-207. doi:10.1590/ S0100-879X1997001000010

9. He P, Cheng X, Staufenbiel M, Li R, Shen Y. Long-term treatment of thalidomide ameliorates amyloid-like pathology through inhibition of $\beta$-secretase in a mouse model of Alzheimer's disease. PLoS One (2013) 8(2):e55091. doi:10. 1371/journal.pone.0055091

10. Chhabra N, Aseri ML, Padmanabhan D. A review of drug isomerism and its significance. Int J Appl Basic Med Res (2013) 3(1):16-8. doi:10.4103/2229-516X. 112233

11. Wade A, Michael Lemming O, Bang Hedegaard K. Escitalopram $10 \mathrm{mg} /$ day is effective and well tolerated in a placebo-controlled study in depression also responsible for the anti-AD effects of (-)-epicatechin in transgenic animals? Otherwise, how may the two stereoisomers have similar efficacy? A good negative control is needed that should be as similar as possible in all experimental set-ups. As commented earlier, one good possibility is taking advantage of enantiomers/stereoisomers. Catechin and epicatechin have identical formula, $\mathrm{C}_{15} \mathrm{H}_{14} \mathrm{O}_{6}$, with four structural possibilities, namely four diastereoisomers. If all four of them act as anti-oxidants, all should display similar efficacy. If they are, however, acting by a specific mechanism, they should have significantly different efficacies. Catechin has a trans configuration and epicatechin has a cis configuration, and each of them has a $(+)$ enantiomer and a (-) enantiomer (Figure 1). We find the possibility of testing these four molecules in equal $\mathrm{AD}$ models, at the same time and by the same experimenter and in a blind way (ideally double blind) of high added value. By providing robust modes of action, the differential effect of stereoisomers in $\mathrm{AD}$ models should help in accelerating translational anti-AD research.

\section{Acknowledgments}

In memoriam of Dr. Adela Mazo and Dr. Anna-María GómezFoix, two colleagues of the Department of Biochemistry and Molecular Biology, who died too young and too soon.

in primary care. Int Clin Psychopharmacol (2002) 17(3):95-102. doi:10.1097/ 00004850-200205000-00001

12. Sicras-Mainar A, Navarro-Artieda R, Blanca-Tamayo M, Gimeno-de la Fuente V, Salvatella-Pasant J. Comparison of escitalopram vs. citalopram and venlafaxine in the treatment of major depression in Spain: clinical and economic consequences. Curr Med Res Opin (2010) 26(12):2757-64. doi:10.1185/03007995. 2010.529430

13. Montgomery SA, Loft H, Sánchez C, Reines EH, Papp M. Escitalopram (Senantiomer of citalopram): clinical efficacy and onset of action predicted from a rat model. Pharmacol Toxicol (2001) 88(5):282-6. doi:10.1034/j.1600-0773. 2001.d01-118.x

14. Bandelow B, Andersen HF, Dolberg OT. Escitalopram in the treatment of anxiety symptoms associated with depression. Depress Anxiety (2007) 24(1):53-61. doi:10.1002/da.20141

15. Porsteinsson AP, Drye LT, Pollock BG, Devanand DP, Frangakis C, Ismail $Z$, et al. Effect of citalopram on agitation in Alzheimer disease: the CitAD randomized clinical trial. JAMA (2014) 311(7):682-91. doi:10.1001/ jama.2014.93

16. Zeng YQ, Wang YJ, Zhou XF. Effects of (-)epicatechin on the pathology of APP/PS1 transgenic mice. Front Neurol (2014) 5:1-10. doi:10.3389/fneur.2014. 00069

17. Ono K, Yoshiike Y, Takashima A, Hasegawa K, Naiki H, Yamada M. Potent antiamyloidogenic and fibril-destabilizing effects of polyphenols in vitro: implications for the prevention and therapeutics of Alzheimer's disease. J Neurochem (2003) 87(1):172-81. doi:10.1046/j.1471-4159.2003.01976.x

Conflict of Interest Statement: The authors declare that the research was conducted in the absence of any commercial or financial relationships that could be construed as a potential conflict of interest.

Copyright (C) 2015 Franco and Petrovic. This is an open-access article distributed under the terms of the Creative Commons Attribution License (CC BY). The use, distribution or reproduction in other forums is permitted, provided the original author(s) or licensor are credited and that the original publication in this journal is cited, in accordance with accepted academic practice. No use, distribution or reproduction is permitted which does not comply with these terms. 